\title{
IDENTIFICACIÓN DE COFACTORES NUTRICIONALES ASOCIADOS A NEOPI.ASIA INTRAEPITELIAL CERVICAI EN MUJERES DE SAN LUIS POTOSÍ
}

\section{IDENTIFICATION OF NUTRITIONAL CO-FACTORS ASSOCIATED WITH CERVICAL INTRAEPITHELIAL NEOPLASIA IN WOMEN FROM SAN LUIS POTOSÍ}

\author{
Omar Medina-De la Cruz, Édgar Villegas-Hinojosa, Darío Gaytán-Hernández, \\ Sandra Olimpia Gutiérrez-Enríquez y Verónica Gallegos-García ${ }^{1 *}$ \\ Fecha de recepción: 6 de julio de 2018 \\ Fecha de aceptación: 12 de septiembre de 2018
}

\begin{abstract}
Resumen - A nivel mundial el Cáncer Cervicouterino ( $\mathrm{CaCu}$ ) es un problema de salud pública. La historia natural de la enfermedad pasa por diferentes grados de neoplasia intraepitelial cervical que modulan su transformación, dependiendo de diversos cofactores relacionados con la alimentación de las pacientes, el sobrepeso y la obesidad, el consumo de suplementos alimenticios y la ingesta de productos light. EI objetivo del presente artículo es identificar esos cofactores nutricionales asociados al desarrollo de neoplasia intraepitelial cervical (NIC) en mujeres de la ciudad de San Luis Potosí, a través del estudio de casos (100) y controles $(300)^{2}$ de pacientes diagnosticadas con distintos grados de NIC por colposcopia, además de que se aplicó un cuestionario validado, se realizó estadística descriptiva e inferencial, así como el cálculo de Odds Ratio (OR). Los resultados indican que los cofactores nutricionales identificados con una significancia estadística fueron la ingesta de refrescos light y de té verde, cuyo consumo se calificó como nutricionales de protección relacionados a la aparición de neoplasia intraepitelial cervical.
\end{abstract}

Palabras clave:

NIC, aspartame, té verde.
Abstract - Cervical Cancer (CeCa) is a public health problem worldwide. The natural history of the disease goes through different degrees of cervical intraepithelial neoplasia that modulate its transformation, depending on various co-factors related to the eating habits of patients, overweight and obesity, the consumption of food supplements and the intake of light products. The objective of this article is to identify those nutritional cofactors associated with the development of cervical intraepithelial neoplasia (CIN) in women from the city of San Luis Potosí, through the study of cases (100) and controls (300) of patients diagnosed with different grades of CIN by colposcopy, in addition to this, a validated questionnaire was applied, descriptive and inferential statistics were carried out, as well as the calculation of Odds Ratio (OR). The results indicate that the nutritional co-factors identified with a statistical significance were the intake of light and green tea soft drinks, as they are linked to the appearance of cervical intraepithelial neoplasia.

\section{Keywords:}

CIN, Aspartame, green tea.

${ }^{1}$ Facultad de Enfermería y Nutrición. Universidad Autónoma de San Luis Potosí, México. Av. Niño Artillero no. 130, Zona Universitaria, CP 78240. *Correo electrónico: vgguaslp2@hotmail.com

2 El grupo de los casos es la población que presenta diferentes grados de neoplasia; el de los controles está compuesto por las pacientes que se encuentran sanas. 


\section{Introducción}

esde 1970, el médico alemán Harald zur Hausen

detectó que el Virus del Papiloma Humano (VPH) puede causar verrugas y distintos cambios a nivel cervical, como neoplasia intraepitelial cervical (NIC), que con las condiciones adecuadas progresa a cáncer cervicouterino (CaCu) (Nour, 2009). Esta enfermedad ocasionó 266 mil defunciones en 2012 y se registraron 528 mil nuevos casos en el mismo año a nivel mundial, por lo que se consideró como el cuarto tipo de cáncer más común que afecta a la población femenina, según la Agencia Internacional para la Investigación del Cáncer (International Agency for Research on Cancer, IARC; Torre, Bray, Siegel, Ferlay, Lortet-Tieulent y Jemal, 2015). En México, de acuerdo con la Organización Mundial de la Salud, en 2008 hubo 10,186 casos nuevos de CaCu (Hernández-Hernández, Apresa-García y Patlán-Pérez, 2015) y para 2010 se registraron 3,864 defunciones por esta causa (AnayaRuiz, Vincent y Pérez-Santos, 2014).

La infección persistente por los tipos virales de VPH de alto riesgo puede provocar desarrollo de NIC, aunque no es suficiente porque se requiere la presencia de otros cofactores de riesgo que se encargan de regular la aparición de la enfermedad, entre los que destacan el inicio de vida sexual a edad temprana, tener múltiples parejas sexuales, uso de anticonceptivos orales, multiparidad, tabaquismo, cocinar con leña, infección por Chlamydia trachomatis, por mencionar algunos de los que ya se tiene evidencia de su relación con el progreso a NIC (Fujii et al., 2013; Poorolajal y Jenabi, 2016; Irimie et al., 2011).

Investigaciones epidemiológicas y de laboratorio han descubierto que algunos cofactores relativos al estado nutricional de las pacientes con NIC tienen un papel fundamental en el progreso de esta enfermedad hacia $\mathrm{CaCu}$; por ejemplo, entre los más estudiados se encuentra la disminución de la actividad física, sobrepeso y obesidad, el consumo de suplementos alimenticios, entre otros (Patel, Pathak, Patel y Sutariya, 2018; Chih, Lee, Colville y Xu, 2013). Además, se ha relacionado la aparición del glioma con la ingesta de refresco light, debido a los compuestos químicos que contiene, como el aspartame (Poorolajal y Jenabi, 2016).

El sobrepeso y la obesidad se definen como una acumulación anormal o excesiva de grasa en el cuerpo que puede repercutir en el estado de salud de las personas (World Health, 2017). Un aumento de grasa corporal se asocia con un mayor riesgo metabólico; su medición es importante, pero requiere de un equipo sofisticado y tiempo, por lo que la medida que se utiliza para predecir el porcentaje de grasa en el cuerpo es el Índice de Masa Corporal (IMF) (Jackson et al., 2002).

Poorolajal y Jenabi (2016) reportaron una asociación positiva entre la obesidad y el CaCu en su metaanálisis, pues se relacionó con un aumento en el riesgo de padecerlo; esto se puede explicar porque el exceso de tejido adiposo altera el metabolismo de hormonas endógenas y la producción de citocinas que tienen que ver con reacciones inflamatorias, y por factores de riesgo genéticos adicionales que pueden promover el crecimiento de células cancerígenas (Gu, Chen y Zhao, 2013). Además, las mujeres con obesidad se someten con menor frecuencia a exámenes de detección de cáncer, en comparación con aquellas que tienen un peso normal, lo cual también refiere a una autoestima baja (Meisinger, Heier y Loewel, 2004).

En las últimas décadas la relación entre la carcinogénesis cervical y la dieta ha recibido mucha atención, debido a la presencia de micronutrientes antioxidantes (carotenoides y tocoferoles) que pueden afectar el progreso de infección por VPH a NIC (Hwang, Kim y Lee, 2010). Se han investigado diversos componentes bioactivos de los alimentos, como las vitaminas y minerales, por su capacidad de modificar el riesgo de cáncer (Greenwald, Anderson, Nelson y Taylor, 2007). La ingesta de suplementos alimenticios como multivitamínicos, vitamina $A$, vitamina $C$, vitamina $E$ y calcio se asocian inversamente con la aparición de NIC (Hwang et al., 2010).

También existen cofactores de protección contra el desarrollo de NIC y CaCu, uno de ellos es el consumo de té 
verde, que contiene antioxidantes polifenólicos como el epigalocatequina-3-Galato (EGCG), agente responsable de su efecto quimiopreventivo. Los antioxidantes de origen natural tienen la capacidad de modular las rutas de transducción de señales celulares, por medio de la activación/represión de factores de transcripción; de tal suerte, pueden interferir en las etapas de iniciación, promoción y progresión de la carcinogénesis (Di Domenico, Foppoli, Coccia y Perluigi, 2012).

En diversos alimentos, principalmente en los refrescos y postres que son bajos en calorías, se utiliza ampliamente el aspartame como edulcorante artificial (Magnuson et al., 2007). El uso de este compuesto fue aprobado por la Administración de Alimentos y Medicamentos de Estados Unidos (Food and Drug Administration), aunque un informe sugiere que el incremento en el número de personas con tumores cerebrales pudiera estar relacionado con su ingesta (Soffritti, Belpoggi, Degli Esposti y Lambertini, 2005). En pacientes con diferentes grados de NIC y CaCu son muy pocos los estudios que se han realizado epidemiológicamente para conocer si este compuesto tiene algún efecto sobre la progresión de la enfermedad. Datos obtenidos in vitro con células cancerosas HeLa expuestas durante $48 \mathrm{~h}$ a diferentes concentraciones de aspartame mostraron cambios en el ARNm de genes implicados en la apoptosis celular, como p53, bax y bcl-2 (Pandurangan et al., 2016); por lo tanto, el objetivo de esta investigación fue identificar cofactores nutricionales y su asociación al desarrollo de NIC en mujeres de la ciudad de San Luis Potosí.

\section{Materiales y métodos}

Se realizó una investigación de casos y de controles en la clínica de colposcopia del Hospital del Niño y la Mujer "Dr. Alberto López Hermosa", durante el periodo junio-diciembre de 2015, la cual previamente fue sometida al comité de ética del hospital y aprobada. La muestra fue de 100 casos diagnosticados con diferentes grados de NIC mediante colposcopia (también se cuenta con resultados de papanicolaou e histopatológico, no publicados) y 300 controles, en los cuales su última citología vaginal fue negativa.

Las pacientes que participaron en este estudio radican en la ciudad de San Luis Potosí, a las que se les pidió que firmaran su consentimiento; para las que eran menores de edad tuvieron que autorizarlo sus tutores; posteriormente se les aplicó un cuestionario con 30 ítems, dividido en dos secciones, la primera pedía datos generales de la unidad de referencia y de la paciente, la segunda se centraba preguntas acerca del factor de riesgo y los cofactores, en donde se incluyeron algunos relacionados con el área nutricional. El instrumento fue confiable, con un Alfa de Cronbach de 0.718 y lo validó una ronda de 10 expertos en el tema (en proceso de publicación).

La información se capturó en el programa estadístico SPSS (por sus siglas del inglés Statistical Package for the Social Sciences) versión 21.0, los datos se analizaron con estadística descriptiva e inferencial y los cofactores se examinaron mediante OR, con una confiabilidad de $95 \%$ y una probabilidad menor a 0.05 .

\section{Resultados y discusión}

En este estudio la muestra fue de 100 casos y de 300 controles, el grupo de los casos fue diagnosticado por colposcopia y se puede observar su distribución en la figura 1. Del grupo de los casos con NIC, 56\% tiene 33 años o menos, lo que indica que las mujeres de la ciudad de San Luis Potosí presentaban algún tipo de neoplasia cervical a edad temprana. Para el grupo de los controles, $61.7 \%$ se ubicaba en el mismo rango de edad (tabla 1). Las infecciones por VPH que progresaron a NIC se encontró en el rango de edad menor a los 35 años (García, 2004). Lo anterior se apoyó en un estudio realizado por López y Lizano (2006) que menciona que una infección por VPH es más frecuente entre los 18 y 30 años. La edad de las pacientes en el grupo de los casos iba de los 19 a 61 años, mientras que para el conjunto de controles era de 16 a 67 años (López y Lizano, 2006). 
En lo que respecta al estado civil de las mujeres con NIC, $69 \%$ eran solteras y $31 \%$ casadas; para las pacientes control, $60.3 \%$ eran solteras y $39.7 \%$ casadas (tabla 1 ). De acuerdo con Eckhardt et al., 2017, la proporción de solteras con $\mathrm{CaCu}$ ha aumentado desproporcionalmente en las últimas cuatro décadas y las investigaciones relacionan este estado civil a la población que tiende a registrar mayor actividad sexual, por lo que es más alta la probabilidad de estar en constante presencia de espermatozoides que pueden actuar como agentes extraños y desencadenar reacciones inflamatorias que a largo plazo provocan el desarrollo de NIC (Romero y Rojas, 2012). Adquirir una infección por VPH (IVPH) que evolucione hacia una NIC no depende totalmente del comportamiento o de la vida sexual de la mujer, es un proceso complejo que implica a su compañero sexual y es importante señalar que el hecho de estar casadas no asegura totalmente su estabilidad sexual (Sepúlveda, González, Napolitano, Roncone y Cavada, 2008).

Algunos estratos sociales están determinados por la pobreza, la desigualdad de género y el escaso acceso a la información sobre la salud, entre otros factores que mantienen a grandes sectores de la población femenina en condiciones de alta vulnerabilidad y riesgo de enfermar por CaCu (Alemán, Bruno, Sánchez, Trujillo y Saucedo, 2012). Del grupo con NIC, 71\% cursó solamente la educación básica o no tenían escolaridad, mientras que para las pacientes control únicamente $30.7 \%$ había cursado el mismo nivel educativo y $38.6 \%$ un grado de educación superior, respecto del $14 \%$ de las pacientes con NIC (tabla 1 ).

Tirado-Gómez et al. (2005) sugieren que niveles escolares y socioeconómicos bajos impiden la detección oportuna de la enfermedad y esto aumenta el riesgo de contraer una IVPH y su avance hacia NIC. Datos de la Encuesta Nacional de la Dinámica Demográfica afirma que aunque las mujeres tuvieron conocimiento acerca de los factores de riesgo para desarrollar CaCu, así como de métodos anticonceptivos, solamente 10.3\% entre 15 y 19 años utilizó algún método anticonceptivo en su primera relación sexual, lo que indica que tener cierto nivel de estudios no significa que se apliquen los conocimientos (López-Carrillo, Suárez-López y TorresSánchez, 2009).

La ocupación entre la población con algún tipo de neoplasia era como sigue: $32 \%$ se dedicaba a labores en el hogar, esto abarca el porcentaje más alto para el grupo de los casos con NIC, frente a $21.7 \%$ en el de los controles, lo que concuerda con la investigación de Romero y Rojas (2012), en la que 43\% de la población en su estudio se dedicaba a las labores domésticas. El CaCu es más frecuente en aquellas mujeres cuya condición económica es baja, esto se relaciona con higiene personal incorrecta y una ingesta deficiente de nutrientes o micronutrientes que llevan a la supresión del sistema inmunológico sistémico o local del moco cervical (Romero y Rojas, 2012).

Acerca del Índice de Masa Corporal (IMC), se utilizó la NOM-008-SSA3-2010 para clasificarlo y se calculó la frecuencia en la población de estudio (tabla 2), en donde se observó que del conjunto de casos con NIC, $61 \%$ presentó un IMC $>25 \mathrm{~kg} / \mathrm{m}^{2}$, mientras que para el grupo de los controles $57.3 \%$ tuvo un IMC $>25 \mathrm{~kg} / \mathrm{m}^{2}$, de ahí que no se observe diferencia significativa entre los dos grupos para la población en estudio de la ciudad de San Luis Potosí. Lo anterior contrasta con la bibliografía en donde se menciona que el sobrepeso y la ingesta de calorías están correlacionadas con el desarrollo de CaCu (Secretaría de Salud, 2010; Choi, Ahn, Park, Cho y Lee, 2017).

El grupo de trabajo de la IARC ha concluido que "la evidencia es demasiado limitada para permitir cualquier conclusión sobre la relación entre el IMC y el riesgo de CaCu" (Vainio, Kaaks y Bianchini, 2002, p. 96). Se ha encontrado que muchas citoquinas y quimioquinas juegan un papel importante en la estimulación del sistema inmune para regular el carcinoma cervical (Feng, 2015), pero para la población del presente estudio en la ciudad de San Luis Potosí no hubo asociación alguna entre el desarrollo de NIC y el sobrepeso u obesidad, aunque se podría aumentar el tamaño de la muestra para observar si este comportamiento se mantiene. 
Para los cofactores nutricionales evaluados, el consumo de refresco light, de suplementos alimenticios y de té verde, se calculó el OR (Odds Ratio) para saber si se podrían considerar como de riesgo o de protección para la población en este estudio. Encontramos que los que tuvieron una significancia estadística fueron la ingesta de refresco light y el consumo de té verde (tabla 3).

El consumo de productos que contienen aspartame se autorizó por la FDA para refrescos en 1983 y se aprobó como edulcorante general en 1996 (Soffritti, Belpoggi, Tibaldi, Degli Esposti y Lauriola, 2007). Su uso se ha relacionado con el riesgo de padecer distintos tipos de cáncer, como el de páncreas, endometrio, gástrico y glioma; sin embargo, para el desarrollo de NIC y su evolución a CaCu la información que existe al respecto es muy limitada (Gallus et al., 2006). El OR que se obtuvo indicó que para esta población en estudio se consideró como un cofactor de protección, lo que difiere de algunos ensayos realizados en ratas Sprague-Dawley al ser sometidas a concentraciones de 2,000 y 4,00 ppm de aspartame (equivalentes al consumo de $100 \mathrm{mg} / \mathrm{kg}$ y $20 \mathrm{mg} / \mathrm{kg}$, respectivamente), los cuales demuestran que estos componentes contribuyen al desarrollo de linfomas y leucemias, así como carcinomas de la glándula mamaria (Rycerz y Jaworska-Adamu, 2013; Lim et al., 2006) .

En otra investigación, Pandurangan et al. (2015) observaron que en células de carcinoma de cuello uterino expuestas a altas concentraciones de aspartame (10 y 20 mM) ocurrieron alteraciones morfológicas importantes; advirtieron que las células eran aplanadas, granulares y con múltiples núcleos en comparación con los controles no tratados (Pandurangan et al., 2016). Cabe señalar que nuestro estudio tiene sesgo, ya que en la aplicación del instrumento no se tomó en cuenta el tiempo durante el cual las pacientes habían ingerido bebidas light (es decir, el tiempo de exposición), la frecuencia ni la cantidad con que las bebían. No obstante, nuestros resultados nos bridan un acercamiento y áreas de oportunidad para realizar futuras indagaciones.
El estado nutricional y el consumo de alimentos pueden ser cofactores importantes que aumentan la persistencia de IVPH y su progresión a $\mathrm{CaCu}$, aunque las pruebas son insuficientes para apoyar la asociación del estado nutricional y la carcinogénesis cervical (García-Cllosas, Castellsagué, Bosch y González, 2005). En lo que respecta a los suplementos alimenticios, al calcular el OR no se encontró ninguna asociación con el desarrollo de NIC, porque el intervalo de confianza (0.71-2.23) no fue estadísticamente significativo. Para la población de estudio este factor no obtuvo relevancia; sin embargo, la literatura señala que la ingesta de suplementos alimenticios, por ejemplo con vitamina C, se asocia a un riesgo dos veces menor de persistencia de VPH, información que sustenta el efecto protector que brindan las frutas ricas en antioxidantes (Siegel et al., 2010).

Lee, Chung, Lee y Ahn (2005) llevaron a cabo una investigación que detectó niveles séricos bajos de vitamina $E$ en pacientes con $\mathrm{CaCu}$; empero, los mecanismos de cómo actúa esta vitamina aún se desconocen, aunque es probable que sus beneficios radiquen en el efecto antioxidante y la capacidad que tiene para eliminar radicales de peróxido de hidrógeno en lípidos y así terminar la reacción en cadena de la peroxidación lipídica. Si los radicales libres y oxidantes no fueran neutralizados por moléculas antioxidantes, los procesos inflamatorios podrían conducir a un daño extenso a las proteínas reguladoras del ADN (Guo et al., 2015). Además, estas moléculas antioxidantes podrían activar la apoptosis al inhibir la ruta de la proteína quinasa C (PKC), promover la función del sistema inmune y frenar el crecimiento de células cancerosas, al disminuir la vía de la fosfoinositida 3-quinasa (Bost, Decoux-Poullot, Tanti y Clavel, 2016)

El consumo de té verde por mujeres de la capital de San Luis Potosí reveló una significancia estadística (0.20-0.51) al realizar la prueba de OR (tabla 3), pero no se cuantificó el tiempo que lo ingirieron. Diversos estudios han demostrado los efectos contra el cáncer del té verde en algunos tipos de tumores malignos, 
como el gástrico, pancreático y colorrectal (Yu et al., 1995). Análisis experimentales demuestran que esta bebida aporta diversos metabolitos como el EGCG, del cual se ha comprobado que bloquea la carcinogénesis e inhibe el crecimiento tumoral tanto in vitro como in vivo, por sus propiedades quimiopreventivas (Ji et al., 1997).

Ahn et al. (2003), en una de las primeras pruebas sobre el efecto del EGCG en la línea de células Caski de CaCu asociada al VPH-16, probaron que su efecto proliferativo depende de la detención del ciclo celular en la fase G1, además de la inducción de apoptosis. Asimismo, Yokoyama, Noguchi, Nakao, Pater e Iwasaka (2004) indicaron que se dirige a la telomerasa e inhibe su actividad, requerida para el inicio y la progresión de las lesiones cervicales (Yokoyama, Noguchi, Nakao, Pater e Iwasaka, 2004; Noguchi et al., 2006). Por su parte, Zou et al. (2010) demostraron una disminución del oncogén E7 del VPH, el aumento de la apoptosis y una inhibición del crecimiento celular con EGCG. De los resultados antes mencionados, está claro que este componente principal del té verde tiene un gran potencial en la prevención y terapia contra el cáncer de cuello uterino (Di Domenico et al., 2012).

\section{Conclusión}

Los estudios en mujeres con NIC y en aquellas sanas con control en la ciudad de San Luis Potosí para conocer cómo influyen cofactores nutricionales en la transformación de las pacientes enfermas hacia CaCu son muy pocos. Por lo tanto, la presente investigación sienta las bases para un acercamiento sobre cómo se comporta esta población, a través de identificar algunos cofactores nutricionales que ofrecen alguna protección contra el desarrollo de esta patología. Para obtener resultados más certeros y con una significancia estadística considerable se requerirá de nuevas investigaciones, en las que el tamaño de la muestra a considerar en el estudio sea mayor y más representativo.
Tabla 1.

Factores sociodemográficos de la población de estudio

\begin{tabular}{|c|c|c|c|c|}
\hline \multirow{2}{*}{} & \multicolumn{2}{|c|}{ CASOS CON NIC } & \multicolumn{2}{c|}{ CONTROLES } \\
\cline { 2 - 5 } & $\mathrm{n}=100$ & $100 \%$ & $\mathrm{n}=300$ & $100 \%$ \\
\hline Edad & \multicolumn{4}{|c|}{} \\
\hline <17 años & 0 & 0.0 & 7 & 2.3 \\
\hline 18-25 años & 17 & 17.0 & 119 & 39.7 \\
\hline 26-33 años & 39 & 39.0 & 59 & 19.7 \\
\hline 34-41 años & 25 & 25.0 & 56 & 18.7 \\
\hline 42-49 años & 14 & 14.0 & 38 & 12.7 \\
\hline 50-57 años & 4 & 4.0 & 10 & 3.3 \\
\hline 58-66 años & 1 & 1.0 & 9 & 3.0 \\
\hline$>67$ años & 0 & 0.0 & 2 & 0.6 \\
\hline Estado civil & \multicolumn{5}{|l}{} & & \\
\hline Solteras & 69 & 69.0 & 181 & 60.3 \\
\hline Casadas & 31 & 31.0 & 119 & 39.7 \\
\hline Escolaridad & \multicolumn{5}{|l}{} \\
\hline Ninguna & 1 & 1.0 & 5 & 1.7 \\
\hline Primaria & 21 & 21.0 & 31 & 10.3 \\
\hline Secundaria & 49 & 49.0 & 56 & 18.7 \\
\hline Preparatoria & 15 & 15.0 & 92 & 30.7 \\
\hline Licenciatura & 10 & 10.0 & 103 & 34.3 \\
\hline Posgrado & 4 & 4.0 & 13 & 4.3 \\
\hline Ocupación & \multicolumn{5}{|l}{} \\
\hline $\begin{array}{c}\text { Labores del } \\
\text { hogar }\end{array}$ & 32 & 32.0 & 65 & 21.7 \\
\hline Autoempleo & 8 & 8.0 & 22 & 7.3 \\
\hline Empleada & 30 & 30.0 & 71 & 23.7 \\
\hline Estudiante & 6 & 6.0 & 106 & 35.3 \\
\hline Profesionista & 24 & 24.0 & 36 & 12.0 \\
\hline
\end{tabular}

Tabla 2.

Índice de masa corporal de la población de estudio

\begin{tabular}{|c|c|c|c|c|c|}
\hline \multirow{2}{*}{ VARIABLES } & \multicolumn{2}{c|}{ CASOS CON NIC } & \multicolumn{2}{c|}{ CONTROLES } \\
\cline { 3 - 6 } & $n=100$ & $100 \%$ & $n=300$ & $100 \%$ \\
\hline IMC & Bajo Peso & 0 & 0.0 & 7 & 2.3 \\
\hline & Peso Normal & 17 & 17.0 & 119 & 39.7 \\
\hline & Sobrepeso & 39 & 39.0 & 59 & 19.7 \\
\hline & Obesidad I & 25 & 25.0 & 56 & 18.7 \\
\hline & Obesidad II & 14 & 14.0 & 38 & 12.7 \\
\hline & Obesidad III & 4 & 4.0 & 10 & 3.3 \\
\hline
\end{tabular}




\section{Tabla 3.}

Cofactores nutricionales en mujeres potosinas

\begin{tabular}{|c|c|c|c|c|}
\hline VARIABLE & $\begin{array}{c}\text { CASOS CON NIC } \\
n=100\end{array}$ & $\begin{array}{c}\text { CONTROLES } \\
n=300\end{array}$ & OR & IC al 95\% \\
\hline \multicolumn{5}{|c|}{ Ingiere refresco light } \\
\hline Sí & 2 & 43 & \multirow{2}{*}{0.200} & \multirow{2}{*}{$0.07-0.513$} \\
\hline No & 98 & 257 & & \\
\hline \multicolumn{5}{|c|}{ Consume suplementos alimenticios } \\
\hline Sí & 21 & 52 & \multirow{2}{*}{1.268} & \multirow{2}{*}{$0.719-2.234$} \\
\hline No & 79 & 248 & & \\
\hline \multicolumn{5}{|c|}{ Ingiere té verde } \\
\hline Sí & 14 & 62 & \multirow{2}{*}{0.510} & \multirow{2}{*}{$0.333-0.870$} \\
\hline No & 86 & 238 & & \\
\hline
\end{tabular}

\section{Gráfica 1.}

Diagnóstico por colposcopia de las pacientes que acudieron al Hospital del Niño y la Mujer "Dr. Alberto López Hermosa" de junio a diciembre de 2015

\section{Grados de neoplasia intraepitelial cervical NIC}

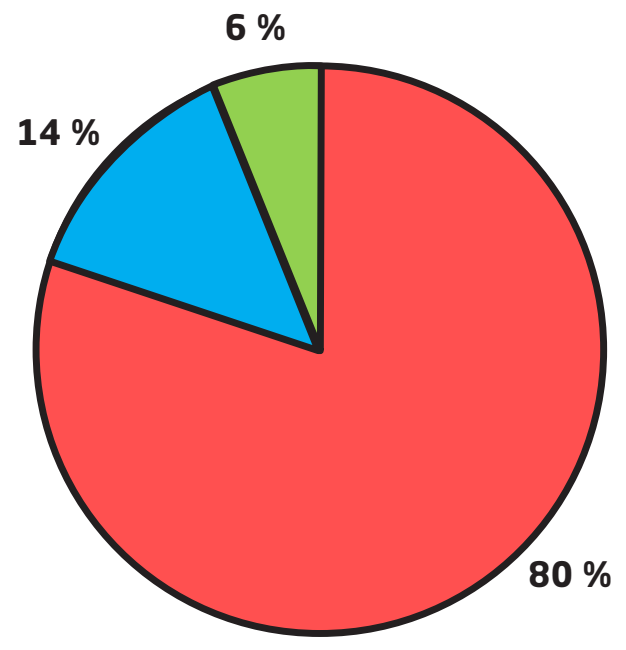

NIC I

NIC II

NIC III

$n=100$ 


\section{Referencias}

Ahn, W. S., Huh, S. W., Bae, S.-M., Lee, I. P., Lee, J. M., Namkoong, S. E., Kim, C. K. y Sin, J.-I. (2003). A major constituent of green tea, EGCG, inhibits the growth of a human cervical cancer cell line, CaSki cells, through apoptosis, G1 arrest, and regulation of gene expression. DNA and cell biology, 22(3), 217-224.

Acevedo, J., Trujillo, M. Á. y López Saucedo, M. L. (coords.). (2012). La problemática de los grupos vulnerables: Visiones de la realidad. México: Universidad Autónoma de Coahuila.

Anaya-Ruiz, M., Vincent, A. K. y Pérez-Santos, M. (2014). Cervical cancer trends in Mexico: incidence, mortality and research output. Asian Pac J Cancer Prev, 15(20), 8689-8692.

Bost, F., Decoux-Poullot, A. G., Tanti, J. F. y Clavel, S. (2016). Energy disruptors: rising stars in anticancer therapy? Oncogenesis, 5(1), e188.

Choi, Y., Ahn, K. J., Park, S. K., Cho, H. y Lee, J. Y. (2017). Adverse effect of excess body weight on survival in cervical cancer patients after surgery and radiotherapy. Radiation oncology journal, 35(1), 48.

Chih, H., Lee, A., Colville, L. y Xu, D. (2013). A review of dietary prevention of Human PapillomavirusRelated Infection of the Cervix and Cervical Intraepithelial Neoplasia. Nutrition and Cancer, 65(3), 317-328.

Di Domenico, F., Foppoli, C., Coccia, R. y Perluigi, M. (2012). Antioxidants in cervical cancer: chemopreventive and chemotherapeutic effects of polyphenols. Biochimica et Biophysica Acta (BBA)-Molecular Basis of Disease, 1822(5), 737-747.
Eckhardt, S., Machida, H., Takiuchi, T., Muderspach, L., Roman, L. y Matsuo, K. (2017). Single marital status among women with malignancy of the uterine cervix in the United States. Gynecologic Oncology, 147(1), 234-235.

Feng, Y.-H. (2015). The association between obesity and gynecological cancer. Gynecology and Minimally Invasive Therapy, 4(4), 102-105.

Fujii, T., Takatsuka, N., Nagata, C., Matsumoto, K., Oki, A., Furuta, R., Maeda, H., Yasugi T., Kawana, K., Mitsuhashi, A., Hirai, Y., Iwasaka, T., Yaegashi, N., Watanabe, Y., Nagai, Y., Kitagawa, T. y Yoshikawa, H. (2013). Association between carotenoids and outcome of cervical intraepithelial neoplasia: a prospective cohort study. International journal of clinical oncology, 18(6), 1091-1101.

Gallus, S., Scotti, L., Negri, E., Talamini, R., Franceschi, S., Montella, M., Giacosa, A., Dal Maso, L. y La Vecchia, C. (2006). Artificial sweeteners and cancer risk in a network of case-control studies. Annals of Oncology, 18(1), 40-44.

García Cllosas, R., Castellsagué, X., Bosch, X. y González, C.A.. (2005). The role of diet and nutrition in cervical carcinogenesis: A review of recent evidence. Int J Cancer, 117, 629-637.

García Arredondo, M. E. (2004). Factores de riesgo de infección por virus del papiloma humano en mujeres derechohabientes de un hospital de seguridad social en San Luis Potosí, SLP junio 2002-julio 2003 (Tesis de maestría). Facultad de Enfermería. Universidad Autónoma de San Luis Potosí. http://ninive.uaslp.mx/jspui/ bitstream/i/2993/6/MSP1FRI00401.pdf

Greenwald, P., Anderson, D., Nelson, S. A. y Taylor, P. R. (2007). Clinical trials of vitamin and mineral 
supplements for cancer prevention. The American journal of clinical nutrition, 85(1), 3145-317S.

Gu, W., Chen, C. y Zhao, K.-N. (2013). Obesityassociated endometrial and cervical cancers. Frontiers in bioscience (Elite edition), 5, 109-118.

Guo, L., Zhu, H., Lin, C., Che, J., Tian, X., Han, S., Zhao, H., Zhu, Y. y Mao, D. (2015). Associations between antioxidant vitamins and the risk of invasive cervical cancer in Chinese women: $A$ case-control study. Scientific reports, 5, 13607.

Hwang, J. H., Kim, M. K. y Lee, J. K. (2010). Dietary supplements reduce the risk of cervical intraepithelial neoplasia. International Journal of Gynecological Cancer, 20(3), 398-403.

Hernández-Hernández, D. M., Apresa-García T. y Patlán-Pérez, R. M. (2015). Panorama epidemiológico del cáncer cervicouterino. Rev Med Inst Mex Seguro Soc,53(1), S154-S161.

Irimie, S., Vlad, M., Mirestean, I. M., Balacescu, O., Rus, M., Balacescu, L. Nagy, V. (2011). Risk Factors in a Sample of Patients with Advanced Cervical Cancer. Applied Medical Informatics, 29(4), 1-10.

Jackson, A. S., Stanforth, P. R., Gagnon, J., Rankinen, T., Leon, A. S., Rao, D. C., Skinner, J.S., Bouchard, C. y Wilmore, J. H. (2002). The effect of sex, age and race on estimating percentage body fat from body mass index: The Heritage Family Study. International journal of obesity and related metabolic disorders: journal of the International Association for the Study of Obesity, 26(6), 789-796.

Ji, B. T., Chow, W. H., Hsing, A. W., McLaughlin, J. K., Dai, Q., Gao, Y. T., Blot, W. J. y Fraumeni, J. F.
(1997). Green tea consumption and the risk of pancreatic and colorectal cancers. International Journal of Cancer, 70(3), 255-258.

Lee, G. J., Chung, H. W., Lee, K. H. y Ahn, H. S. (2005). Antioxidant vitamins and lipid peroxidation in patients with cervical intraepithelial neoplasia. Journal of Korean medical science, 20(2), 267-272.

Lim, U., Subar, A. F., Mouw, T., Hartge, P., Morton, L. M., Stolzenberg-Solomon, R., Campbell, D., Hollenbeck, A. R. y Schatzkin, A. (2006). Consumption of aspartame-containing beverages and incidence of hematopoietic and brain malignancies. Cancer Epidemiology and Prevention Biomarkers, 15(9), 1654-1659.

López, A. y Lizano, M. (2006). Cáncer cervicouterino y el virus del papiloma humano: la historia que no termina. Cancerología, 1(1), 31-55.

López-Carrillo, L., Suárez-López, L. y TorresSánchez, L. (2009). Detección del cáncer de mama en México: síntesis de los resultados de la Encuesta Nacional de Salud Reproductiva. Salud pública de México, 51, s345-s349.

Magnuson, B. A., Burdock, G. A., Doull, J., Kroes, R. M., Marsh, G. M., Pariza, M. W., Spencer, P. S., Waddell, W. J., Walker, R.y Williams, G. M. (2007). Aspartame: a safety evaluation based on current use levels, regulations, and toxicological and epidemiological studies. Critical reviews in toxicology, 37(8), 629-727.

Meisinger, C., Heier, M. y Loewel, H. (2004). The relationship between body weight and health care among German women. Obesity, 12(9), 14731480.

Noguchi, M., Yokoyama, M., Watanabe, S., Uchiyama, M., Nakao, Y., Hara, K. e Iwasaka, T. (2006). Inhibitory 
effect of the tea polyphenol,(-)-epigallocatechin gallate, on growth of cervical adenocarcinoma cell lines. Cancer letters, 234(2), 135-142.

Nour, N. M. (2009). Cervical cancer: a preventable death. Reviews in Obstetrics and Gynecology, Z(4), 240-244.

Patel, A., Pathak, Y., Patel, J. y Sutariya, V. (2018). Role of nutritional factors in pathogenesis of cancer. Food Quality and Safety, 2(1), 27-36.

Pandurangan, M., Enkhtaivan, G., Mistry, B., Chandrasekaran, M., Noorzai, R. y Kim, D. H. (2016). Investigation of role of aspartame on apoptosis process in HeLa cells. Saudi journal of biological sciences, 23(4), 503-506.

Poorolajal, J., y Jenabi, E. (2016). The association between BMI and cervical cancer risk: a metaanalysis. European Journal of Cancer Prevention, 25(3), 232-238.

Romero L., K. P., y Rojas G., J. (2012). Frecuencia de los factores de riesgo de Cáncer Cervicouterino en mujeres de 14-65 años, Comunidad Ramadas, Provincia Tapacarí-Cochabamba gestión 2012. Revista Científica Ciencia Médica, 15(1), 18-21.

Rycerz, K. y Jaworska-Adamu, J. E. (2013). Effects of aspartame metabolites on astrocytes and neurons. Folia neuropathologica, 51(1), 10-17.

Secretaría de Salud. (2010). NOM-008-SSA3-2010 Para el tratamiento integral del sobrepeso y la obesidad. México: Autor.

Sepúlveda, P., González, F., Napolitano, C., Roncone, E. y Cavada, G. (2008). Cáncer de cuello uterino: sobrevida a 3 y 5 años en Hospital San José. Revista chilena de obstetricia y ginecología, 73(3), 151-154.
Siegel, E. M., Salemi, J. L., Villa, L. L., Ferenczy, A., Franco, E. L. y Giuliano, A. R. (2010). Dietary consumption of antioxidant nutrients and risk of incident cervical intraepithelial neoplasia. Gynecologic oncology, 118(3), 289-294.

Soffritti, M., Belpoggi, F., Degli Esposti, D. y Lambertini, L. (2005). Aspartame induces lymphomas and leukaemias in ratsa L'aspartame induce linfomi e leucemie nei ratti. Eur. J. Oncol, 10(2), 107-116.

Soffritti, M., Belpoggi, F., Tibaldi, E., Degli Esposti, D. y Lauriola, M. (2007). Life-span exposure to low doses of aspartame beginning during prenatal life increases cancer effects in rats. Environmental Health Perspectives, 115(9), 1293.

Tirado-Gómez, L. L., Mohar-Betancourt, A., LópezCervantes, M., García-Carrancá, A., Franco-Marina, F. y Borges, G. (2005). Factores de riesgo de cáncer cervicouterino invasor en mujeres mexicanas. Salud pública de México, 47(5), 342-350.

Torre, L. A., Bray, F., Siegel, R. L., Ferlay, J., LortetTieulent, J., y Jemal, A. (2015). Global cancer statistics, 2012. CA: a cancer journal for clinicians, 65(2), 87-108.

Vainio, H., Kaaks, R. y Bianchini, F. (2002). Weight control and physical activity in cancer prevention: international evaluation of the evidence. European journal of cancer prevention: the official journal of the European Cancer Prevention Organisation (ECP), 11, S94-S100.

World Health, 0. (2017). Obesity and Overweight factsheet from the WHO. Autor.

Yokoyama, M., Noguchi, M., Nakao, Y., Pater, A., e Iwasaka, T. (2004). The tea polyphenol,(-)epigallocatechin gallate effects on growth, apoptosis, 
and telomerase activity in cervical cell lines. Gynecologic oncology, 92(1), 197-204.

Yu, G. P., Hsieh, C. C., Wang, L. Y., Yu, S. Z., Li, X.-I. y Jin, T. H. (1995). Green-tea consumption and risk of stomach cancer: a population-based case-control study in Shanghai, China. Cancer Causes \& Control, 6(6), 532-538.
Zou, C., Liu, H., Feugang, J. M., Hao, Z., Chow, H. H. S. y García, F. (2010). Green tea compound in chemoprevention of cervical cancer. International journal of gynecological cancer: official journal of the International Gynecological Cancer Society, 20(4), 617.

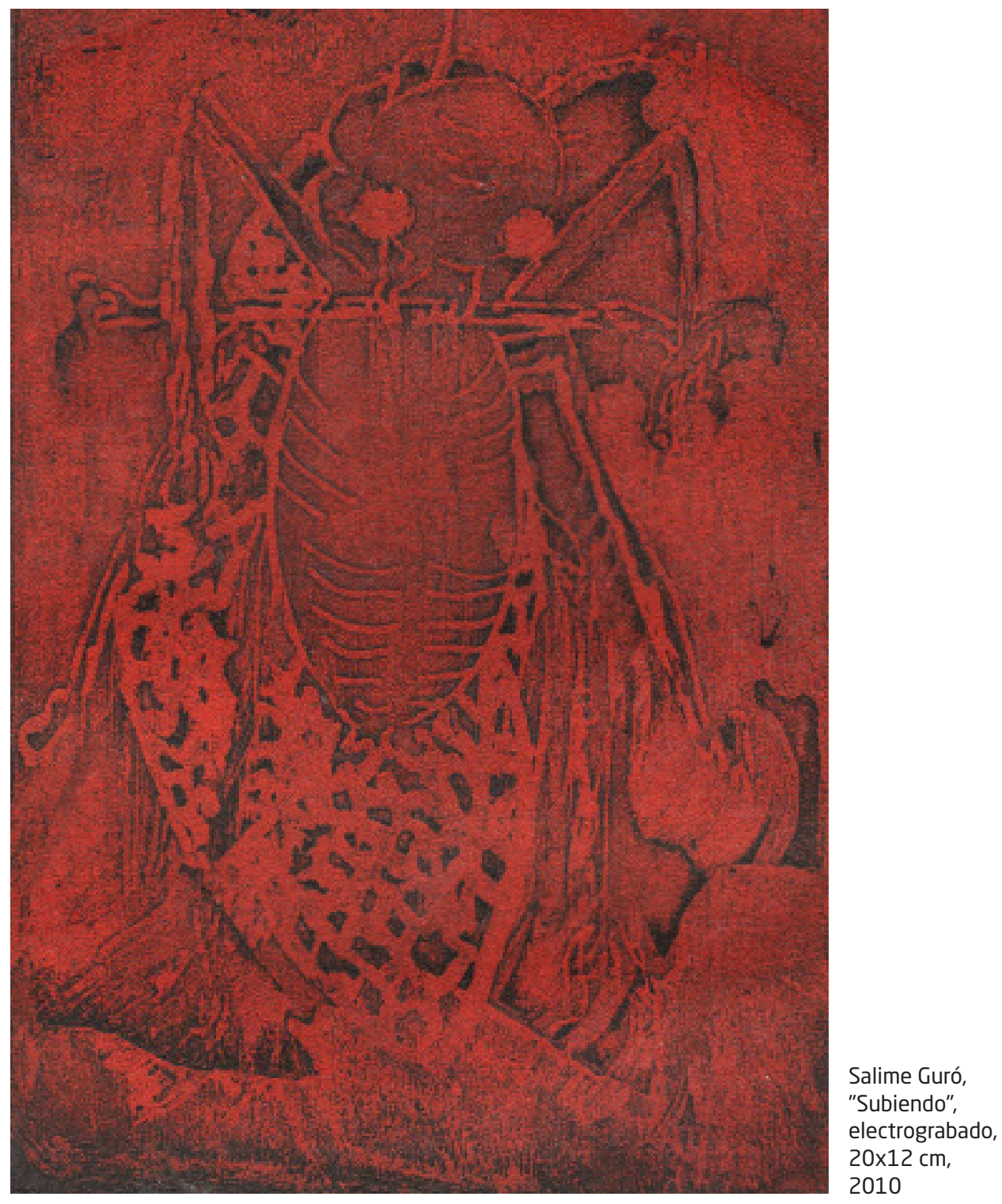




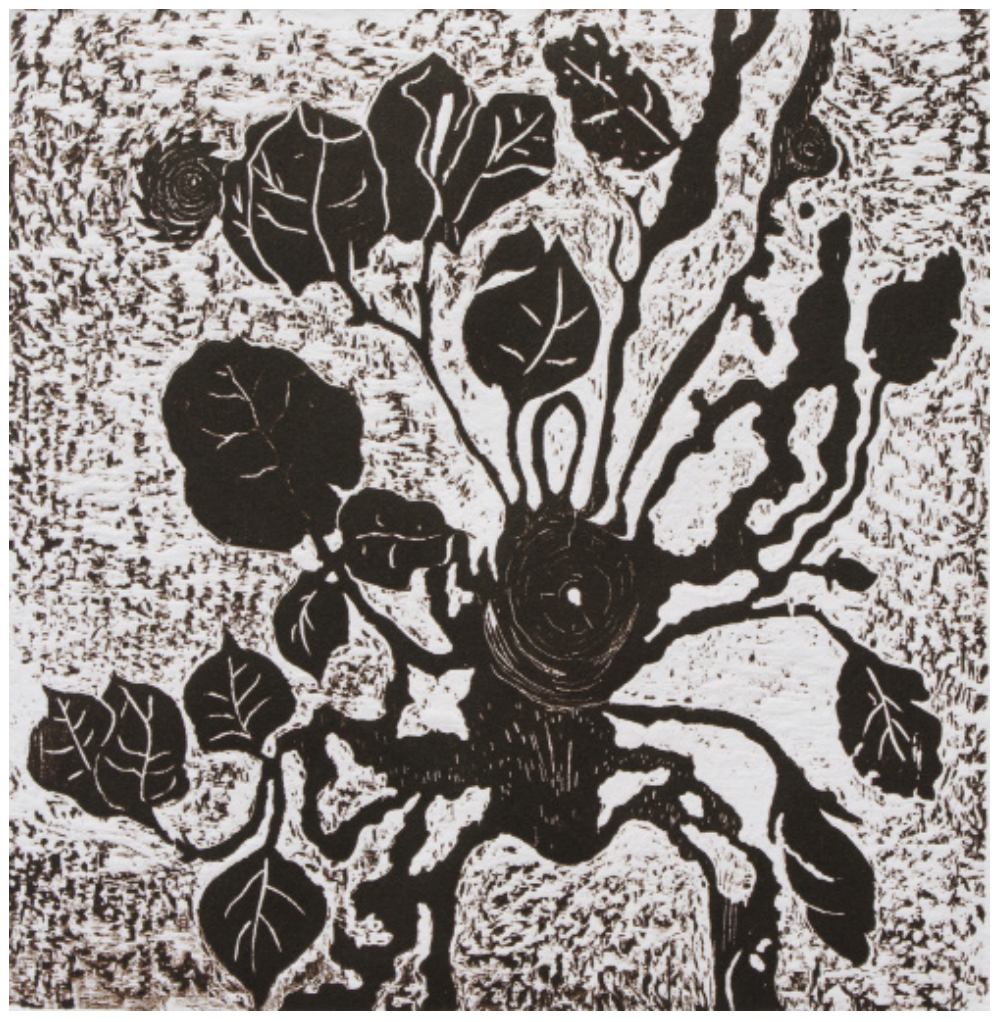

Soledad

Vázquez,

"Bugambilia",

xilografía,

$24 \times 23 \mathrm{~cm}$

2018

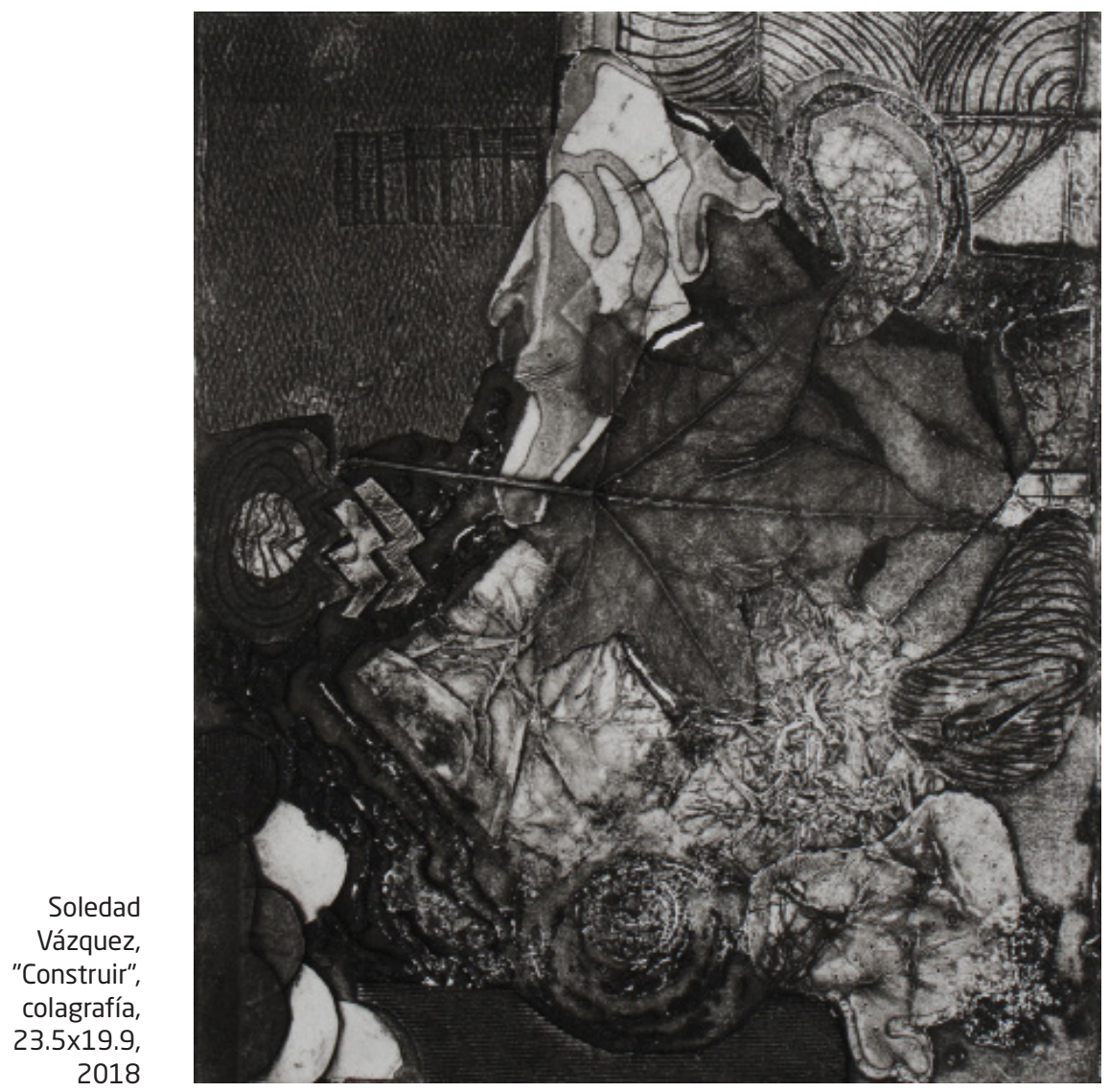

Tequio, septiembre-diciembre 2018, vol. 2, no. 4 